\title{
Exploiting flow to control the in vitro spatiotemporal distribution of microbubble-seeded acoustic cavitation activity in ultrasound therapy
}

5

\author{
Antonios N Pouliopoulos, Simone Bonaccorsi and James J Choi \\ Noninvasive Surgery and Biopsy Laboratory, Bioengineering Department, \\ Imperial College London, London, SW7 2AZ, United Kingdom \\ E-mail: j.choi@imperial.ac.uk
}

10

Keywords: ultrasound therapy; acoustic cavitation; acoustic emissions; microbubbles; passive cavitation detection; drug delivery; capillary opening 


\begin{abstract}
Focused ultrasound and microbubbles have been extensively used to generate therapeutic bioeffects. Despite encouraging in vivo results, there remains poor control of the magnitude and spatial distribution of these bioeffects due to the limited ability of conventional pulse shapes and sequences to control cavitation dynamics. Thus current techniques are restricted by an efficacy-safety trade-off. The primary aim of the present study was to incorporate the presence of flow in the design of new short pulse sequences, which can more uniformly distribute the cavitation activity. Microbubbles flowing (fluid velocity: $10 \mathrm{~mm} / \mathrm{s}$ ) through a $300 \mu \mathrm{m}$ tube were sonicated with a focused $0.5 \mathrm{MHz}$ transducer while acoustic emissions were captured with an insert focused 7.5MHz passive cavitation detector, with the two foci being co-axially aligned and overlapped. Whereas conventional sequences are composed of a long burst $(>10,000$ cycles) emitted at a low burst repetition frequency $(<10 \mathrm{~Hz})$, we decomposed this burst into short pulses by adding intervals to facilitate inter-pulse microbubble movement. To evaluate how this sequence influenced cavitation distribution, we emitted short pulses (peak-rarefactional pressure (PRP): 40-366kPa, pulse length (PL): 5-25 cycles) at high pulse repetition frequencies (PRF: $0.625-10 \mathrm{kHz}$ ) for a burst length of $100 \mathrm{~ms}$. Increased cavitation persistence, implied by the duration of the microbubble acoustic emissions, was a measure of improved distribution due to the presence of flow. Sonication at lower acoustic pressures, longer pulse intervals and lower PLs improved the spatial distribution of cavitation. Furthermore, spectral analysis of the microbubble emissions revealed that the improvement at low pressures is due to persisting stable cavitation. In conclusion, new short-pulse sequences were shown to improve spatiotemporal control of acoustic cavitation dynamics during physiologically relevant flow. This could lead to adjustable distribution of the generated in vivo bioeffect and therefore efficient and safe treatment of a wide range of pathologies.
\end{abstract}




\section{Introduction}

Recent advances in ultrasound therapy involve the use of focused ultrasound (FUS) and systemically administered microbubbles (MBs) (Stride and Coussios 2010) for a range of therapeutic applications such as targeted drug delivery (Ferrara et al 2007), sonothrombolysis

5 (de Saint Victor et al 2014), sonoporation (Yu and Xu 2014), renal ultrafiltration (Deelman et al 2010) and blood-brain barrier opening (Konofagou 2012). FUS has the advantage of penetrating deep into the body and localising its interaction with MBs within a well-defined target region, i.e. the focal volume. MBs, which are typically composed of a gas-core and a lipid-shell, can be driven into radial oscillations by the rarefactional and compressional phases of the acoustic wave (Apfel 1997). This acoustically driven behaviour, known as acoustic cavitation, has been shown capable of producing either therapeutic or harmful bioeffects. Thus, understanding how cavitation dynamics are dictated by ultrasound parameters and designing new pulse shapes and sequences to produce new dynamics could improve therapeutic and safety outcomes.

Specific characteristics of an ultrasonic pulse shape (i.e. centre frequency, pulse length (PL), and peak-rarefactional pressure (PRP)) and sequence (i.e. pulse repetition frequency (PRF)) have been shown to dictate specific types and magnitudes of cavitation. The centre frequency influences the frequency that MBs radially oscillate, with the oscillation amplitude being the greatest for MBs of resonant size at low acoustic pressures (Marmottant et al 2005, Apfel and Holland 1991, Lauterborn 1976). The PRP determines the amplitude of radial oscillation while the PL matches with the cavitation duration. PLs also influence the primary radiation force (Dayton et al 2002, 1997); long pulses push the MBs across a greater length, because the force is applied over a longer duration (Palanchon et al 2005). Modification of these three pulse shape features can produce a range of cavitation behaviours from low-amplitude non-inertial cavitation to high-amplitude inertial cavitation, in which MBs expand and collapse violently due to the inertia of the surrounding fluid (Apfel 1997). Pulse shapes trigger cavitation dynamics on a time scale of microseconds and therefore interact with a single population of MBs. In therapeutic ultrasound, a large portion of the MBs are destroyed or modified by the end of the pulse and thus a pulse-off-time is required to allow new MBs to replenish the target volume. Conventional therapeutic pulse sequences thus employ a pulse repetition frequency (PRF) typically on the order of $\mathrm{Hz}$ and a 
defined number of pulses, which determines the number of cavitation events the tissue is treated by and the duration of the treatment.

Depending on the ultrasonic pulse shape and the properties of the fluid surrounding the MBs, ultrasound exposure can cause the MBs to radially oscillate in either linear or non-linear cavitation modes: stable and non-inertial, stable and inertial, or transient and inertial cavitation (Church and Carstensen 2001). Cavitation modes can be identified by analysing the acoustic emissions from the MBs. High magnitude non-inertial and low magnitude inertial cavitation events radiate acoustic emissions at the driving ultrasound frequency and its integer multiples or harmonics (Apfel 1997). In contrast, high magnitude inertial cavitation, which is created by the unstable expansion and subsequent violent MB collapse (Chomas et al 2001) yields broadband acoustic emissions. Spectral analysis of the acoustic emissions can thus identify distinct cavitation dynamics.

Inertial and non-inertial cavitation modes have been shown to cause bioeffects by exerting mechanical forces on biological interfaces, such as the vascular wall or the cell membrane. Theoretical and experimental approaches have characterized MB-vessel interactions according to the mechanical stress exerted on the vascular wall and have correlated these measurements to vascular damage (Hosseinkhah et al 2013, Hosseinkhah and Hynynen 2012, Chen et al 2012, Caskey et al 2007, Qin and Ferrara 2006). Bubble collapse was shown to exert up to four times higher stress than stable bubble expansion (Hosseinkhah et al 2013). Optical evidence suggested that the stress caused by stable cavitation originates from the direct contact between the oscillating MBs and the endothelium of small vessels (Caskey et al 2007). Although the exact mechanisms remain unclear, MB-mediated mechanical agitation of the vasculature has been shown to alter capillary permeability in the brain (Hynynen et al 2001), kidney (Li et al 2013, Fischer et al 2009), liver (Wang et al 2013), prostate (Liu et al 2013) and other organs. The advantage of these capillary opening techniques is that they can noninvasively and locally increase the delivered concentration of drugs to a target tissue, thus reducing the required systemic doses and thereby minimizing deleterious side-effects. Drug delivery is particularly limited in the brain due to the blood-brain barrier (BBB) which tightly separates the vessel lumen from the brain interstitial space (Zlokovic 2008). Neurodegenerative diseases like Alzheimer's, Parkinson syndrome and multiple sclerosis remain untreatable partly because of the impermeability of the BBB to large therapeutic molecules such as antibodies or peptides (Konofagou 2012). For this reason, a large body of 
work has focused on the disruption of BBB using the FUS-MBs system (Wang et al 2014, Nhan et al 2013, Vlachos et al 2011, Choi et al 2011b, Tung et al 2010). Yet it has been previously shown that conventional pulse shapes and sequences are limited by an efficacysafety trade-off. Higher pressures increased doses delivered (Baseri et al 2010), but they were also associated with cell necrosis or erythrocyte extravasations (Daffertshofer et al 2005, Hynynen et al 2001).

Conventional ultrasound pulse shapes and sequences can control the type and magnitude of cavitation, but they are inefficient in distributing cavitation in physiological systems (Choi and Coussios 2012). In capillary opening, cavitation-induced permeability increases are produced as spot-like patterns resulting in an inhomogeneous distribution of a model drug (Choi et al 2007b, Stieger et al 2007). This spatial inhomogeneity in treatment could be due to cavitation activity being poorly distributed throughout the microvasculature. Long PLs (>10ms) immobilize flowing MBs upon their entrance into the focal volume due to primary radiation forces (Dayton et al 2002), thus constraining their activity to a limited region (Choi and Coussios 2012). Typical therapeutic pulses have durations of 10-100ms and pulse repetition frequencies (PRFs) on the order of $\mathrm{Hz}$ (Nhan et al 2013, O'Reilly and Hynynen 2012, Choi et al 2011a, Hynynen et al 2001), yet the highest magnitude and number of cavitation events occur within the first few milliseconds, thus producing an inefficient and heterogeneous treatment. In vivo studies have shown that long PLs lead to heterogeneous drug distributions, with molecules being accumulated near large vessels, such as arterioles and venules (Choi et al 2010). In contrast, short PLs emitted at repetition periods on the order of microseconds were shown to enhance the desired in vivo bioeffect without compromising safety (O'Reilly et al 2011, Choi et al 2011b). Further complicating the ability to control cavitation is that the pressure threshold for inertial cavitation decreases with the PL (Radhakrishnan et al 2013, Gruber et al 2014) and thus long PLs promote faster MB destruction. Additionally, high acoustic pressures and the concomitant inertial cavitation events introduce greater spatial bias on the cavitation distribution (Choi and Coussios 2012). For clinical applications, conventional ultrasonic pulses could produce over-treated and under-treated areas within the same focal volume, resulting in unsafe or inefficient therapies, respectively.

The primary aim of the present study is to exploit flow in the design of a new therapeutic ultrasonic pulse sequence that will improve acoustic cavitation distribution over time and 
space, while avoiding or minimising damage-related cavitation activity. We evaluated the use of short PLs interspaced throughout a 100ms burst with off-time intervals placed between. The use of this novel burst sequence was empirically shown to facilitate improved drug delivery distribution in vivo (Choi et al 2011b, 2011a), however there has been no direct evidence of improved cavitation distribution. The main assumption is that sonication of a MB population during flow using our burst sequence would enable MBs to move during the short "off" state of the sequence. The distance MBs travel would depend on the time intervals between two adjacent pulses and the fluid velocity. During the "on" state, MBs are expected to be immobilised after a few cycles due to primary radiation force effects pushing them against the tubular (or vascular) wall in the direction of the FUS propagation. If MBs remain intact during each pulse, the spatial distribution of MB activity could be adjusted solely by modifying the idle intervals between FUS pulses. Whereas conventional pulse shapes and sequences can control the cavitation type, magnitude and duration, our new burst sequence has the additional control of cavitation distribution.

\section{Methods}

\subsection{MB manufacturing}

In-house manufactured lipid-shelled MBs were prepared and characterized prior to every experiment. Three phospholipids (Avanti Polar Lipids Inc., AL, USA), namely Dipalmitoylphosphatidylcholine (DPPC-82\%), Dipalmitoylphosphatidic acid (DPPA-8\%), and dipalmitolyphosphatidylethanolamine-PEG5000 (DPPE-PEG5000-10\%) were mixed and diluted with saline and glycerol. Perfluorobutane (FluoroMed L.P., Texas, USA) was added and the mixture was then amalgamated. The MB concentration and size distribution were measured using a haemocytometer and a microscope following previously described protocols (Sennoga et al 2010). The MB concentration was found to be $7.3 \pm 4 \cdot 10^{9} \mathrm{MBs} / \mathrm{ml}$ and the mean size $1.62 \pm 0.87 \mu \mathrm{m}$ while excluding bubbles with diameters below $0.5 \mu \mathrm{m}$. In order to ensure clinical relevance, the MB suspension was diluted to $\sim 5 \cdot 10^{7} \mathrm{MBs} / \mathrm{ml}$, approximately $25 \mathrm{x}$ the acceptable dose for clinical applications which is $0.02 \mathrm{ml} / \mathrm{kg}$ (Unger et al 2004) or $2.04 \cdot 10^{6} \mathrm{MBs} / \mathrm{ml}$. This calculation was performed for an average male mass of $70 \mathrm{~kg}$ with a total blood volume of $5 \mathbf{L}$. 


\subsection{Experimental setup}

Sonication of flowing MBs was performed in an in vitro system at physiologically relevant fluid velocities (figure 1). A 300- $\mu \mathrm{m}$ diameter silicone elastomer tube (Saint-Gobain Performance Plastics, Paris, France) was submerged and positioned horizontally in a tank containing deionised and degassed water. MBs were injected into the tube using a syringe pump (Instech, Plymouth Meeting, PA, USA) which allowed a constant flow rate of $43.6 \mu \mathrm{l} / \mathrm{min}$, corresponding to fluid velocity of $10 \mathrm{~mm} / \mathrm{s}$. This velocity was on the same order of magnitude as the peak blood velocity in a medium-sized arteriole of size comparable to our tube model (Tangelder et al 1986).

A $0.5 \mathrm{MHz}$ spherical-segment, single-element transducer (active diameter: 64mm, nominal f-number: 0.98, nominal FWHM: $5.85 \mathrm{~mm}$, focal length: 62.6mm, part number: H107; Sonic Concepts Inc., Bothel, WA, USA) was used to emit the FUS. The tube was placed $6 \mathrm{~cm}$ away from the transducer's surface and overlapped its focal point, which was determined earlier using a $0.2 \mathrm{~mm}$ diameter PVDF needle hydrophone (Precision Acoustics Ltd,

15 Dorchester, UK). Ultrasound emission and detection was controlled with Matlab (The Mathworks, Natick, MA, USA). Two function generators (33500B Series, Agilent technologies, Santa Clara, CA, USA) were employed for the generation of the various pulse shapes/sequences, with the first defining the pulse sequence and the second dictating the pulse shape. The signal was amplified by a $50 \mathrm{~dB}$ power amplifier (Precision Acoustics Ltd, Dorchester, UK) and applied to the $0.5 \mathrm{MHz}$ transducer via a matching box (Sonic Concepts Inc., WA, USA). 

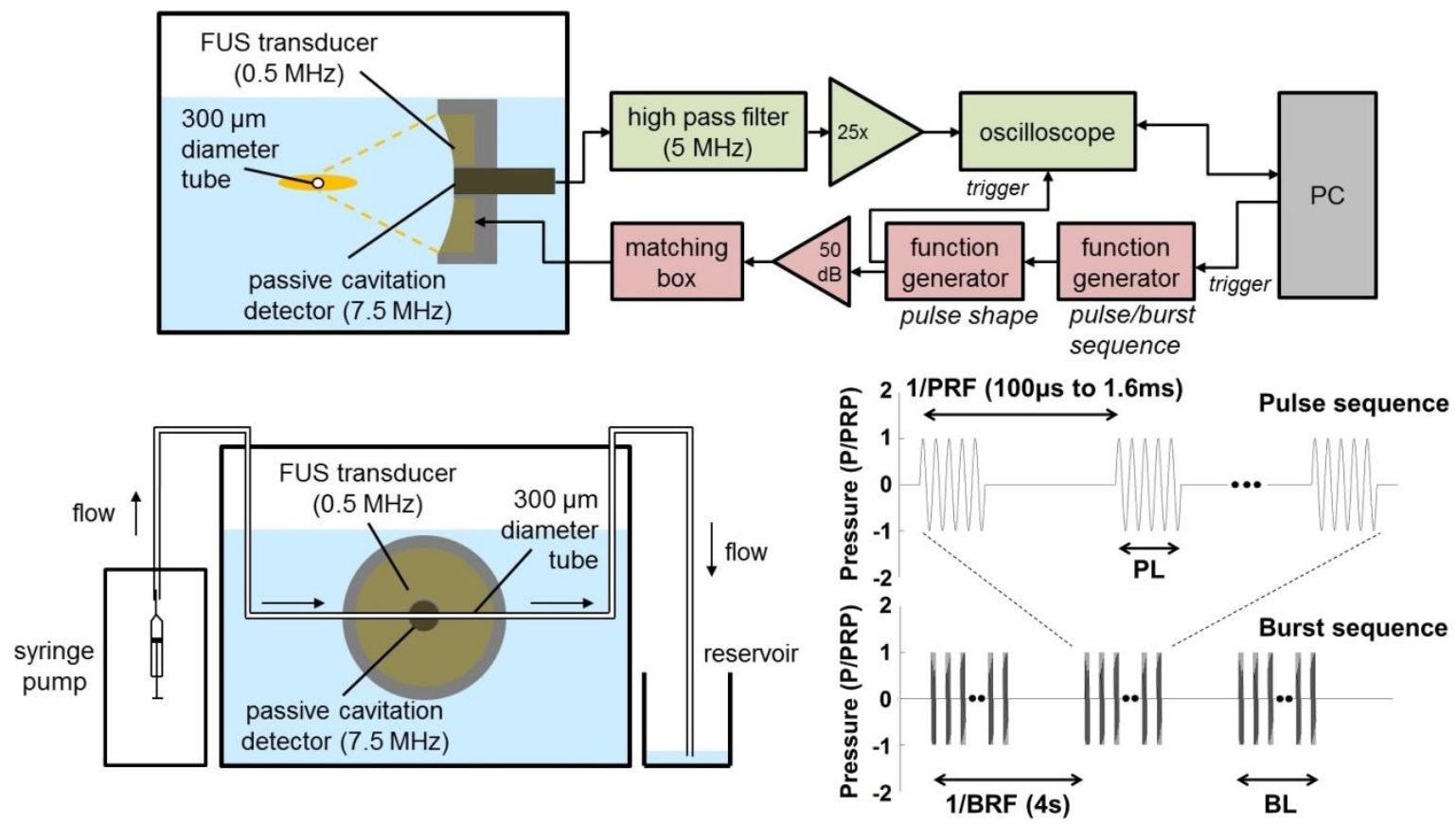

Figure 1. Experimental setup. (a) Two function generators were used to produce distinct pulse shapes and sequences emitted by the $0.5 \mathrm{MHz}$ FUS transducer. The PCD signal was filtered with a $5 \mathrm{MHz}$ high pass filter, captured with an oscilloscope and saved to a PC for processing. (b) A syringe pump kept a constant MB flow rate within the $300-\mu \mathrm{m}$ diameter tube. (c) Each pulse of ultrasound was emitted at a specific pulse repetition frequency for a limited duration specified by a burst length. Each burst was emitted at a specific burst repetition frequency. Abbreviations: PRP: peak rarefactional pressure; PL: pulse length; PRF: pulse repetition frequency; BL: burst length; BRF: burst repetition frequency.

MB acoustic response was captured with a $7.5 \mathrm{MHz}$ transducer (diameter: $\mathbf{1 2 . 7} \mathbf{m m}$, nominal

f-number: 4.72, focal length: 60mm, part number: U8423589; Olympus Industrial, Essex, UK) coaxially aligned with the $0.5 \mathrm{MHz}$ transducer. In order to avoid signal saturation due to reflection and scattering by the tube at the fundamental frequency, the PCD signal was fed through a 5-MHz analog high pass filter $\left(\mathbf{f}_{\mathbf{c}}=5 \mathrm{MHz},-\mathbf{- 2 0 d B}\right.$ at $4 \mathrm{MHz}, 500 \mathrm{hm}$, part number: MH-500P-C-P; Allen Avionics, Mineola, NY, USA) and a $28 \mathrm{~dB}$ preamplifier (Stanford

Research Systems, Sunnyvale, CA, USA) to an oscilloscope (8-bit, sampling rate $\mathbf{5 0 M S a / s}$; Tektronix, Bracknell, UK). The time-domain trace was then saved to a PC for processing.

\subsection{Ultrasound parameters}

The design of efficient pulse sequences was based on optimising specific parameters of the emitted ultrasound. Optimisation was performed in terms of spatiotemporal distribution of the cavitation energy emissions. Based on previous in vivo results (Choi et al 2011b, 2007a), we refined the parametric search to a small subset of values (Table 1). As discussed, the parameters used differ from conventional pulse sequences in the time scale. Whereas conventional pulse repetition periods are on the order of seconds, here the time interval 
between the pulses is on the order of microseconds and milliseconds (figure 1). Additionally, conventional pulse lengths are often circa 100ms. For this reason, a 100ms-long continuous wave pulse was used as the control sequence.

Table 1: Ultrasound parameters

$\begin{array}{lcc}\text { Peak rarefactional pressure }(\mathrm{kPa}) & 40-366 \\ \text { Pulse repetition frequency }(\mathrm{kHz}) & 0.62,1.25,2.5,5 & 1.25,2.5,5,10 \\ \text { Pulse length (no. of cycles) } & 5 & 25 \\ \text { Burst length (ms) } & 100 \\ \text { Burst repetition frequency }(\mathrm{Hz}) & 0.25 \\ \text { Number of bursts } & 10\end{array}$

Burst length was kept constant for all the experiments $(B L=100 \mathrm{~ms})$, since we wanted to compare the energy emission distribution in various pulse sequences within a defined period of ultrasound exposure. Bursts were emitted at a burst repetition frequency (BRF) of $0.25 \mathrm{~Hz}$, i.e. every 4 seconds, to allow MB replenishment into the focal volume. Meanwhile, we tested

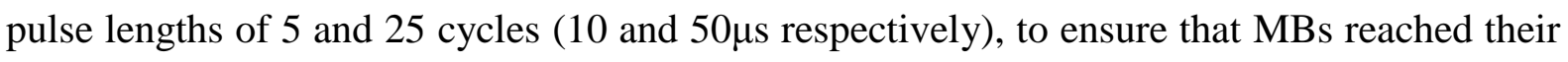
highest oscillation amplitude and mode of cavitation at a given pressure (Church 2005). For each distinct set of parameters, a total of 10 consecutive bursts were emitted.

\subsection{Data analysis}

The collected raw data were processed in Matlab. Analysis was conducted in terms of energy emission and spectral content.

\subsubsection{Energy analysis}

Cavitation magnitude, duration and distribution were determined by analysing the energy emitted by the cavitating MBs. Energy emissions were calculated by integrating the time domain signal with respect to time (i.e. integration of the squared voltage vs. time curve). The energy values correlate with the PCD's electrical energy, which is proportional to the acoustic energy emitted by the MB cavitation activity. For the purpose of this work, "energy" 
is relevant as a comparative measurement across the sequences rather than as an absolute value.

Furthermore, we corrected the calculated energy values to compensate for the electrical noise contribution. The mean noise energy value was calculated in a signal segment where acoustic cavitation was absent and then subtracted from the energy value of each point throughout the $100 \mathrm{~ms}$ duration. Cumulative energy plots were created to characterise the temporal distribution of the cavitation emissions. Two distinct times were calculated, $\mathrm{t}_{50}$ and $\mathrm{t}_{80}$, which represented the time that energy emissions reached $50 \%$ and $80 \%$ of the maximum energy. Increased time constants inferred longer MB movement, because the presence of flow and pulse intervals enables the MB population to move a greater distance along the longitudinal axis of the tube, thus producing a more uniform cavitation distribution along the lateral axis.

In addition to increased cavitation persistence, the pulse sequence should be able to produce a desired magnitude of cavitation, since this has been previously correlated to distinct bioeffects such as drug delivery increase (Choi et al 2014, Graham et al 2014), tissue heating (Jensen et al 2012) or hemolysis (Chen et al 2003). We therefore defined a parameter, the acoustic cavitation output, which is calculated as:

$$
\text { Output }=E_{\text {total }} \cdot t_{80}(1)
$$

Increased levels of this parameter suggest that the pulse sequences efficiently produce cavitation of both high magnitude (or mechanical stress through MB-vessel mechanical interactions) and increased persistence. Cavitation output of the pulse sequences was used as an additional comparison metric to assess their potential bioeffect, regarding not only the cavitation magnitude but also its spatiotemporal distribution.

\subsubsection{Spectral analysis}

Acoustic emissions were spectrally analysed to determine the cavitation mode and duration, both throughout a $100 \mathrm{~ms}$ burst and across distinct pulses of a burst. For the latter method, each pulse was windowed and analysed independently.

In order to quantify the spectral features, we calculated the Harmonic to Broadband (HtB) ratio. A high $\mathrm{HtB}$ value would indicate the dominance of harmonic traits while a $\mathrm{HtB}$ value close to 1 would indicate the dominance of broadband emissions. The frequency domain was 
separated into two spectral regimes: a) the harmonic component, i.e. the region surrounding each harmonic peak $\left(f_{H, n} \pm 0.1 M H z, n \in[10,20]\right)$, and b) the non-harmonic component, i.e. the region between the harmonic peaks $\left(f_{H, n}+0.1 M H z, f_{H, n+1}-0.1 M H z\right)$. Harmonic frequencies are given by $f_{H, n}=n f_{0}$ where $f_{0}=0.5 \mathrm{MHz}$. The spectral energy within the

5 harmonic and non-harmonic regions was summed and division of the harmonic value by the non-harmonic value gave the $\mathrm{HtB}$ ratio. In order to compensate for the different bandwidth of the two regions $(0.2 \mathrm{MHz}$ for the harmonic and $0.3 \mathrm{MHz}$ for the nonharmonic), the harmonic energy was weighted by a factor of $3 / 2$, so that equal spectral magnitudes for the two regimes would result to a $\mathrm{HtB}$ ratio of 1.

\section{Results}

\subsection{Energy analysis}

MB acoustic emissions were used to calculate the cavitation energy generated during each ultrasound burst. As expected, the mean total cavitation energy (averaged across the 10 consecutive bursts) rose with the PRP (figure 2). The minimum pressure for detecting cavitation activity with our system was approximately $100 \mathrm{kPa}$. Any MB activity below this threshold was not detectable owing to the low signal-to-noise ratio (SNR). The energy of the received emissions from pulsed excitation followed a linear trend with increasing pressure in all the sequences evaluated, while the continuous wave control reached a plateau after $250 \mathrm{kPa}$, which was in good agreement with previous studies (Choi and Coussios 2012). There was no clear dependence of the PRF on the magnitude of the total burst energy. For the PL of 5 cycles there was no notable difference between the various PRFs (figure 2(a)), which was unexpected if we take into account the different duty cycles or the different number of emitted pulses of each sequence. Lower PRFs (i.e. $0.62 \mathrm{kHz}$ and $1.25 \mathrm{kHz}$ ) led to lower burst energies for the PL of 25 cycles (figure 2(b)), since a lower number of pulses were emitted during the $100 \mathrm{~ms}$ burst. The $2.5 \mathrm{kHz}$ pulse sequence generated higher energy emissions compared to the other PRFs (for pressures above $200 \mathrm{kPa}$ ), an effect which was previously observed for pulsed sonication (Flynn and Church 1984, Ciaravino et al 1981). 

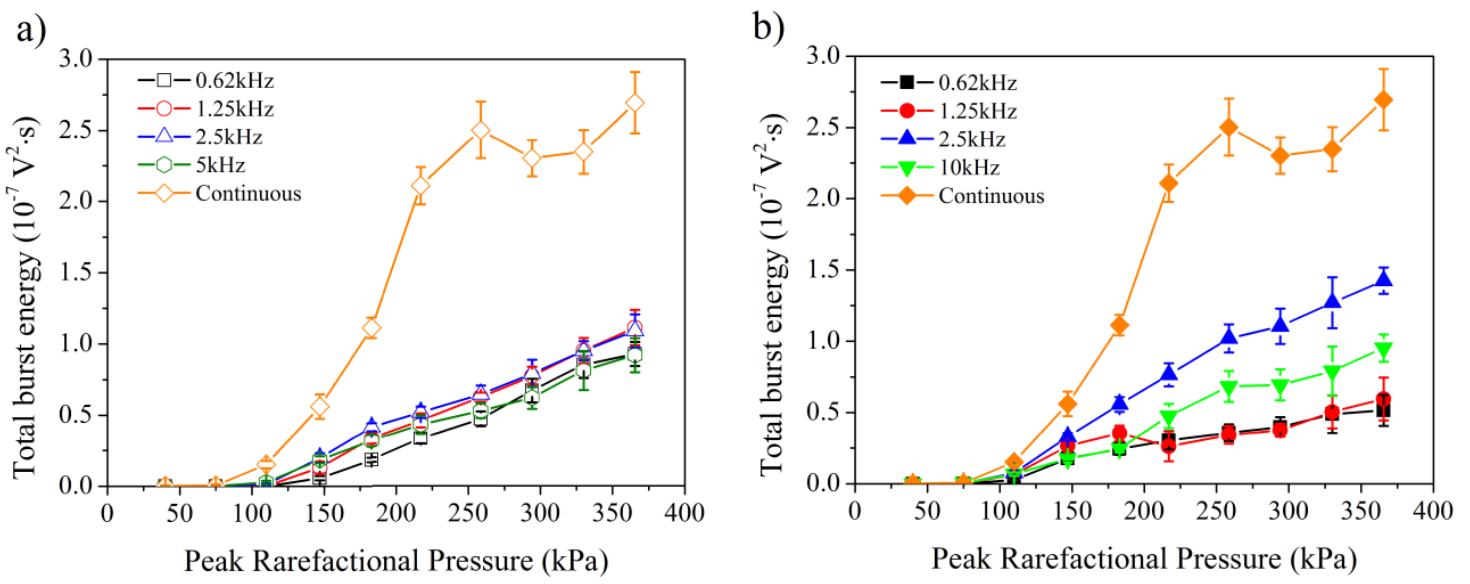

Figure 2. Total burst energies (mean \pm S.D.) for the various pulse sequences as a function of PRP for PL of 5 (a) and 25 cycles (b). Averaging was performed across the 10 consecutive bursts.

Whereas the total burst energies provided insight into the magnitude of cavitation activity within the focal volume, we also sought to characterize the spatiotemporal distribution of this activity by analysing features of the cumulative energy over time (figure 3). Within a 100ms burst sonication, the cumulative energy rose rapidly in the first few milliseconds and then plateaued. In nearly all the evaluated sequences, there was no further increase after the first $40 \mathrm{~ms}$, thus indicating a superfluous pulse-on time beyond this point (figure 3(a)). The stepwise increase depicts the "on" and "off" states of the sonication, with each step's width matching the duration of a single pulse. We assumed that slower rise times of the cumulative energy are related to improved spatial distribution of cavitation activity since MBs are flowing. Following normalisation to the total burst energy, the $t_{50}$ and $t_{80}$ constants, or the time needed for energy emissions to reach $50 \%$ and $80 \%$ of the total value, were calculated (figure 3(a)). Lower acoustic pressures had higher time constants for both 5 and 25 cycle PLs and for all PRFs (figures 3(b)-(e)), providing evidence for prolonged cavitation activity. Moreover, comparison between 5 (figures 3(b) and 3(d)) and 25 cycles (figures 3(c) and 3(e)) revealed consistently higher $\mathrm{t}_{50}$ and $\mathrm{t}_{80}$ values for the shorter PL. Unlike 25-cycle pulses, short 5-cycle pulses increased and stabilised the $t_{50}$ and $t_{80}$ values for higher pressures (PRP>200kPa). It is also evident that lower PRFs (or long pulse repetition periods) led to higher time constants, since the same number of pulses is emitted in a longer period of time. An exception to this trend is a PRF of $0.62 \mathrm{kHz}$ which produced lower time constants than $1.25 \mathrm{kHz}$ at low pressures and a PL of 25 cycles (figures 3(c) and 3(e)). This may originate from the relatively low achieved SNR in the corresponding time-domain signal and/or inefficient noise correction. As previously discussed we excluded values below $100 \mathrm{kPa}$ for 
all data sets and data points of $\mathrm{PRP}=110 \mathrm{kPa}(\mathrm{PRF}=0.62 \mathrm{kHz}$ and $1.25 \mathrm{kHz})$ for the $\mathrm{PL}$ of 5 cycles due to low SNR.

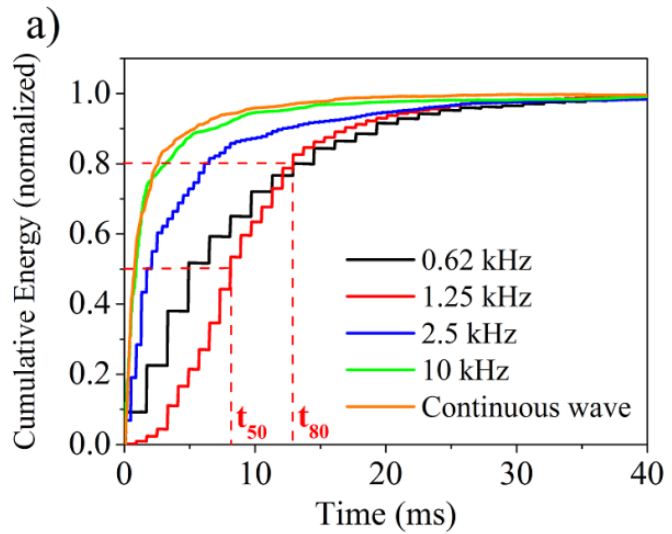

b)

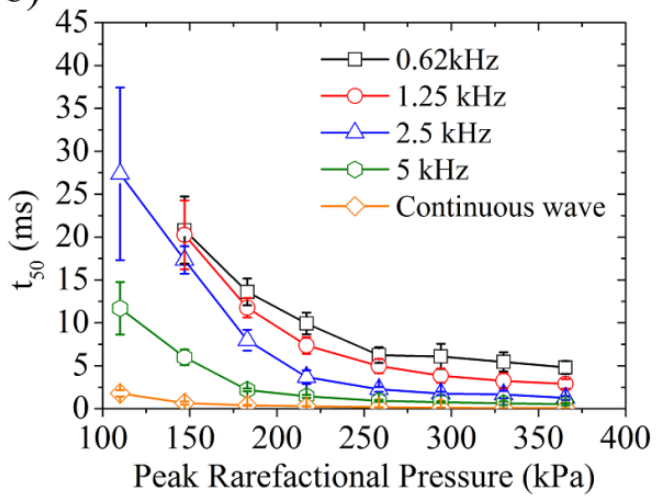

d)

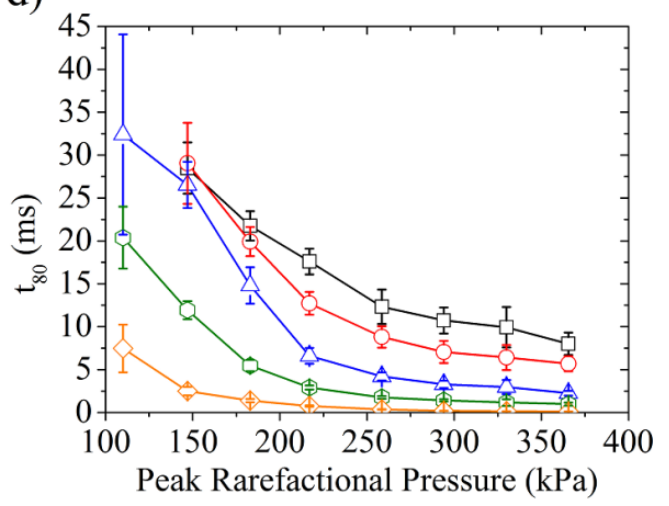

c)

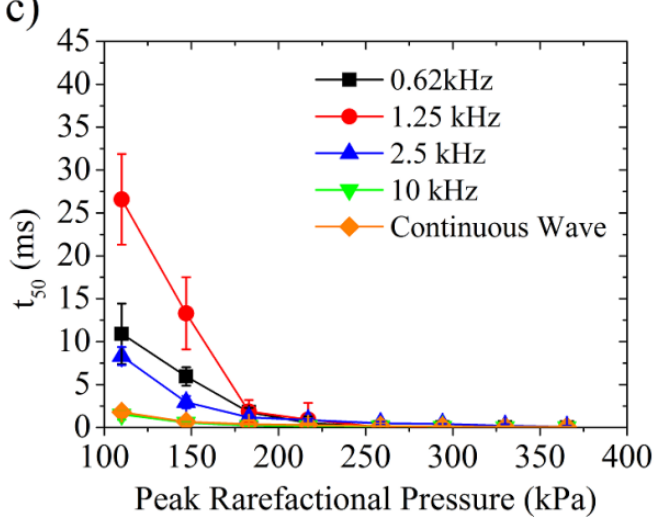

e)

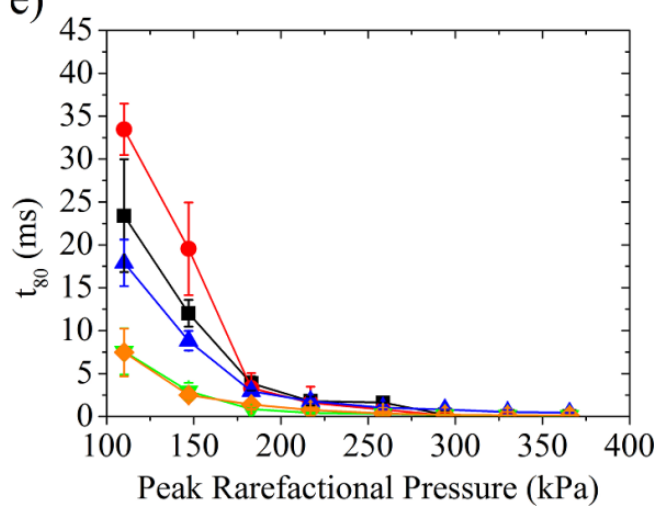

Figure 3. Temporal distribution of cavitation activity. (a) Normalised cumulative energy ( $\mathrm{PRP}=147 \mathrm{kPa}, \mathrm{PL}=25$ cycles) with representative $t_{50}$ and $t_{80}$ constants. $t_{50}(b, c)$ and $t_{80}(d, e)$ values (mean \pm S.D.) indicate the time until energy reaches the $50 \%$ and the $80 \%$ of its maximum value for PL of 5 (b,d-empty symbols) and 25 cycles (c,efilled symbols). Averaging was performed across the 10 consecutive bursts.

Efficient therapeutic pulse sequences should ideally produce an adjustable magnitude of uniformly distributed cavitation activity. Quantification of the combination of energy emissions with high temporal persistence was achieved with the calculation of the cavitation output parameter using equation (1). Essentially, cavitation output (figure 4) is derived by 
multiplying the data from figures 2 (a) and 2(b) by the data from figures $3(\mathrm{~d})$ and $3(\mathrm{e})$ respectively. Output was consistently higher for low PRFs for pulses of 5 cycles but there is no visible trend across the PRPs (figure 4(a)). In contrast, for the 25 cycles case it is maximized within the PRP range of $100-200 \mathrm{kPa}$ (figure 4(b)), confining the region where both burst energy and acoustic cavitation duration are high enough. It can be deducted that lower PRPs around the peak of $150 \mathrm{kPa}$ are the most suitable candidates for the optimal 25 cycle pulse sequence (figure 4(b)). Output is larger at higher pressures (circa $225 \mathrm{kPa}$ ) in the continuous wave control. Generally 5-cycle pulses resulted in higher values than 25 cycles, which is reasonable if we account for the increased $t_{80}$ values of the 5-cycle sequences (figures 3(d) and 3(e)) and the energy values which are on the same order of magnitude (figure 2).
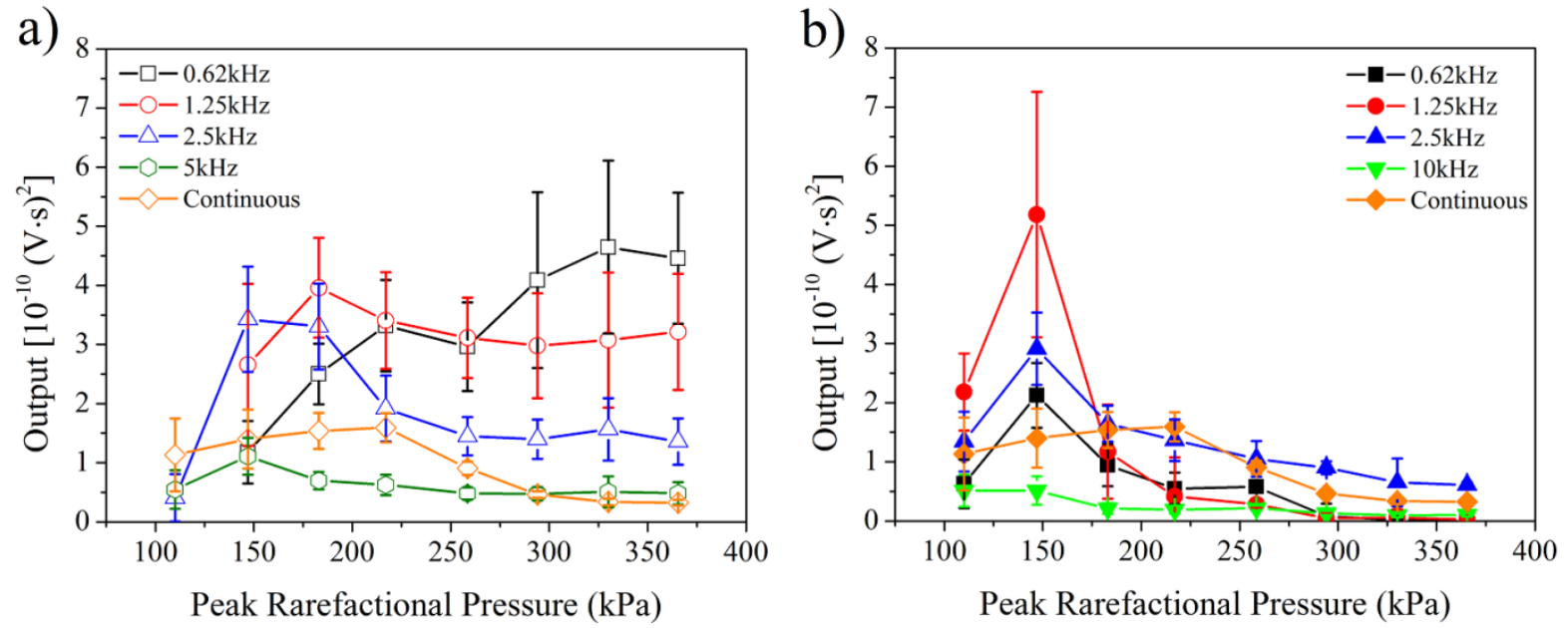

Figure 4: Acoustic cavitation output. Output values (mean \pm S.D.) as a function of PRP for PL of 5 (a) and 25 cycles (b). Averaging was performed across the 10 consecutive bursts.

\subsection{Spectral analysis}

In addition to the analysis of the emitted MB energy, we were interested in identifying the cavitation mode through spectral analysis. Fourier Transforms of the PCD signal acquired during the entire ultrasound burst had similar structures to one another, presenting peaks at the harmonics to the driving frequency (an example is given in figure 5 (a) for PRP $=147 \mathrm{kPa}$, $\mathrm{PL}=25$ cycles). The bandwidth of the harmonics was greater for the evaluated pulse sequences than for the continuous wave control, due to the shorter PL (5 and 25 cycles). Furthermore, their magnitude varied with the PRF. The HtB ratio was highest in the region of $P R P=100$ $200 \mathrm{kPa}$ (figure 5(b)), indicating increased dominance of the harmonic element, i.e. higher levels of stable cavitation rather than transient and inertial cavitation. Qualitative 
interpretation of the spectra confirmed that $\mathrm{HtB}$ ratios decrease above $200 \mathrm{kPa}$ due to the ascent of the broadband signal floor, i.e. due to increased levels of transient inertial cavitation.

a)

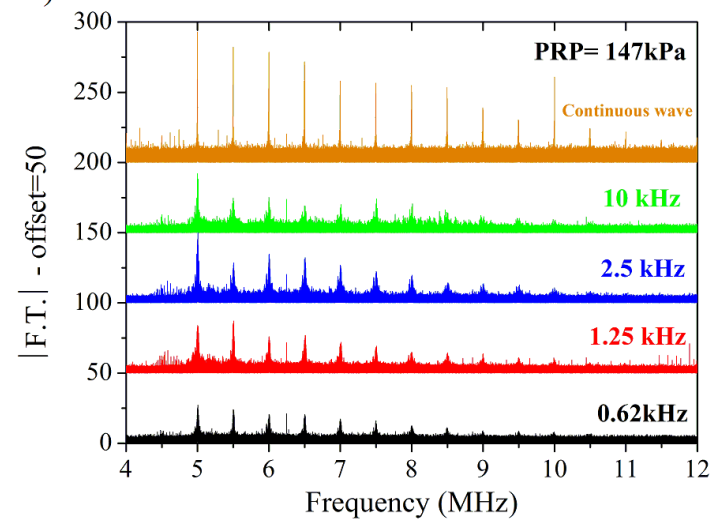

b)

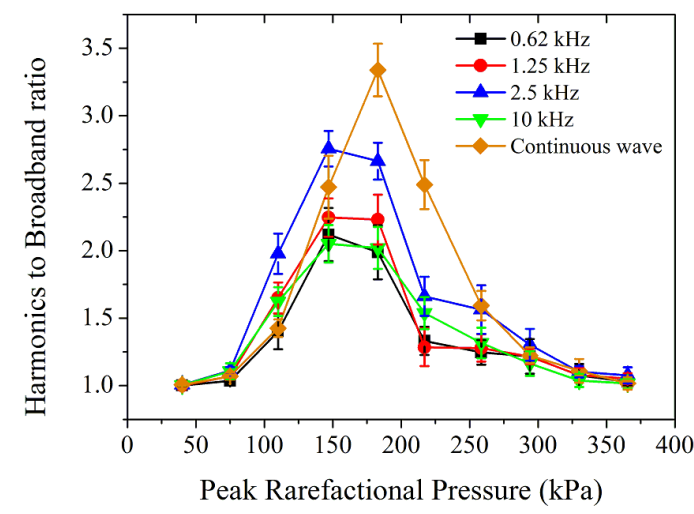

5 Figure 5. Spectral analysis of the PCD signal acquired during the entire ultrasound burst. a) Magnitude of the Fourier Transforms for different PRFs (PRP $=147 \mathrm{kPa}, \mathrm{PL}=25$ cycles). b) Harmonic to Broadband ratio (mean \pm S.D.) vs. PRP for various pulse sequences ( $P L=25$ cycles). Averaging was performed across the 10 consecutive bursts.

Pulse-by-pulse analysis revealed the time-resolved cavitation mode (figure 6). At low pressures (figure 6(a)) there was no detectable MB activity and at high pressures (figure 6(c)) short-duration transient cavitation was detected. Moderate pressures maintained cavitation activity for a longer period than higher pressures (figure 6(b)) and induced mainly stable oscillations which were indicated by the persistence of harmonics and limited presence of broadband signal. Traces of transient cavitation during the first pulses of moderate pressures were detected (figure 6(b)) which could be attributed to the destruction of resonant MBs. Quantification of the spectral characteristics of the 25-cycle pulse sequences is given in figure 7. Here the time-resolved $\mathrm{HtB}$ ratio obtain its maximum values in the pressure region of $100-200 \mathrm{kPa}$. Interestingly, with the exception of the $0.62 \mathrm{kHz}$ sequence (figure $7(\mathrm{a})$ ), the dominance of harmonic over the non-harmonic component persists for approximately 40 pulses (figures 7(b)-(d)). The broad spectral bandwidth of the short 5-cycle pulse sequences rendered the accurate quantification of the $\mathrm{HtB}$ ratio difficult and thus was not processed here. However, it was expected that a similar trend was present in both the 5- and 25-cycle cases. 

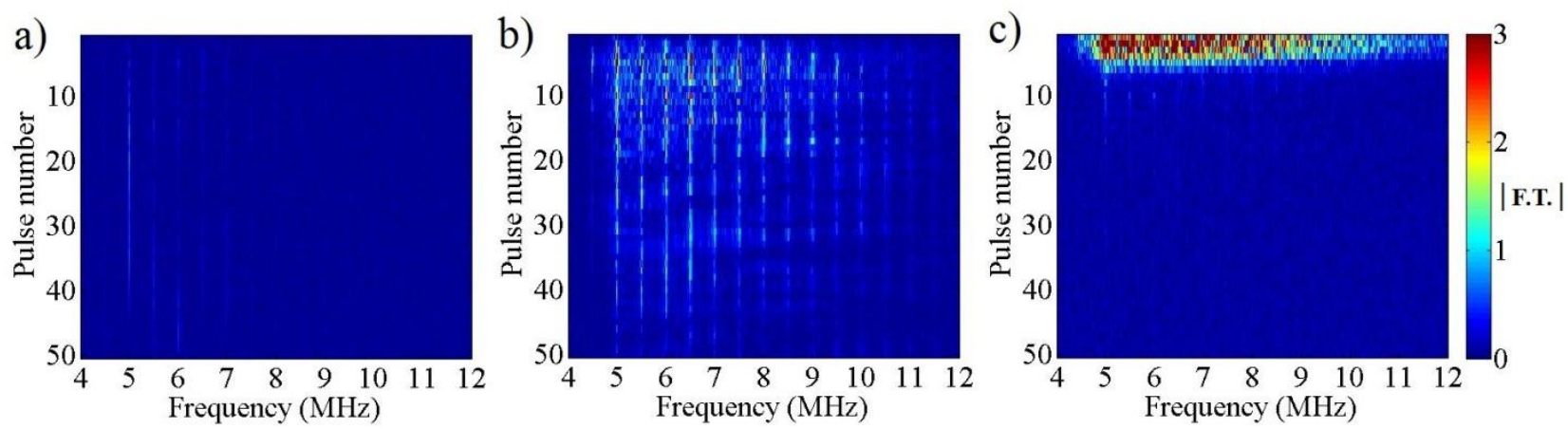

Figure 6: Pulse-by-pulse spectral analysis. Magnitude of Fast Fourier Transforms for PRP of $75 \mathrm{kPa}(\mathrm{a}), 147 \mathrm{kPa}$ (b) and $294 \mathrm{kPa}(\mathrm{c}) . \mathrm{PRF}=2.5 \mathrm{kHz}, \mathrm{PL}=25$ cycles.

a) PRF: $0.62 \mathrm{kHz}$ Time (ms)

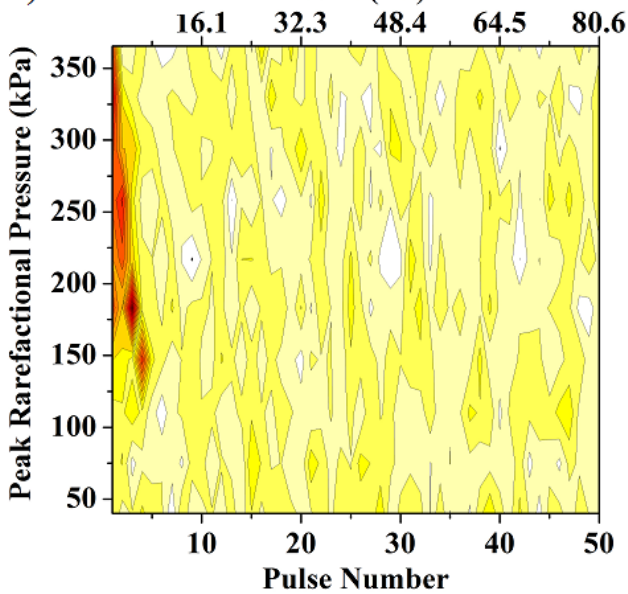

c) PRF: $2.5 \mathrm{kHz}$ Time (ms)

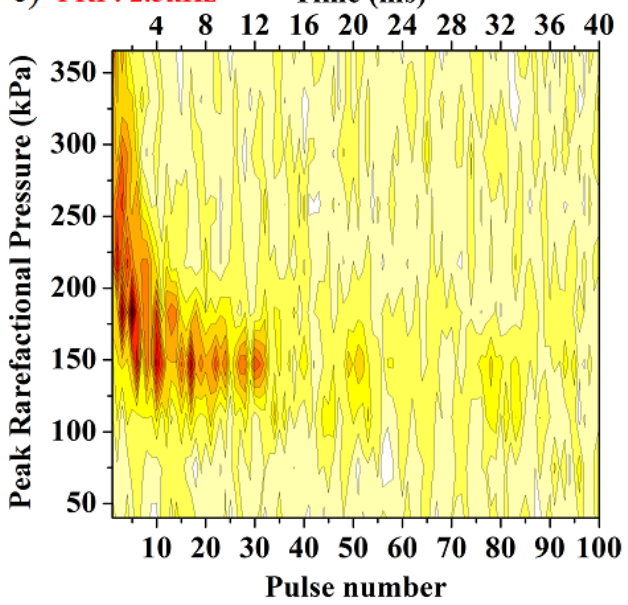

b) PRF: $1.25 \mathrm{kHz}$ Time (ms)

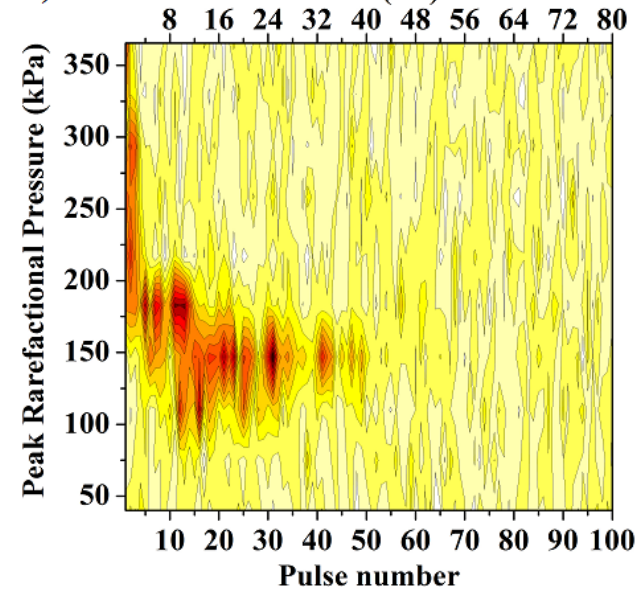

d) PRF: $10 \mathrm{kHz}$ Time (ms)

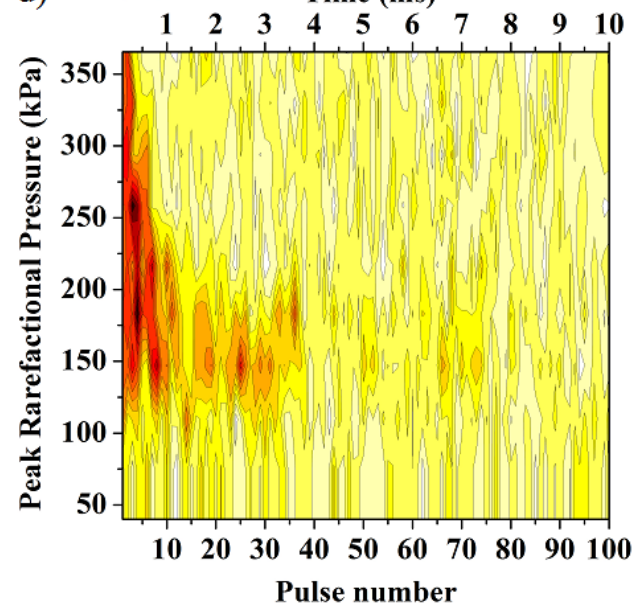

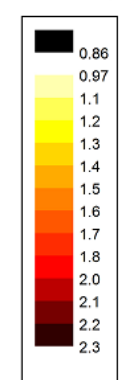

Figure 7. Extrapolated contour plots of the pulse-by-pulse HtB ratios for PRFs of $0.62 \mathrm{kHz}(\mathrm{a}), 1.25 \mathrm{kHz}$ (b), 2.5 $\mathrm{kHz}(\mathrm{c})$ and $10 \mathrm{kHz}(\mathrm{d}) . \mathrm{PL}=25$ cycles. 


\section{Discussion}

\subsection{Acoustic cavitation distribution}

We have demonstrated that the spatiotemporal distribution of acoustic cavitation activity seeded by flowing MBs can be adjusted by modifying specific features of an ultrasonic pulse shape and sequence. Whilst in previous therapeutic approaches high acoustic pressures and transient events enhanced the desired bioeffect, e.g. the vascular permeability increase (Nhan et al 2013, Choi et al 2011a, O'Reilly and Hynynen 2012, Choi et al 2010, 2007a), herein moderate pressures and stable activity of the cavitation nuclei is shown to facilitate longer and more uniform cavitation distribution (figure 6). It is known that increased pressures above the threshold of transient inertial cavitation lead to rapid collapse of MBs and to a concomitant high energy output (figure 1), i.e. increased induced shear stress (Caskey et al 2007). The exerted force in this case is higher but dispersed across the vasculature (Choi et al 2007b, Stieger et al 2007) and proportional to the MB concentration in the vessel. Furthermore, the fast elimination of cavitation nuclei in these pressures (figure 6) reduces cavitation longevity and necessitates longer pulse repetition periods (on the order of 0.1 to 2 seconds) to allow MB replenishment in the focal volume (Choi et al 2011a). Previous studies have determined the pressure threshold of transient inertial cavitation initiation in the area above 250-300kPa, both in high (Radhakrishnan et al 2013) and low (Gruber et al 2014) insonation frequencies. As a result, it can be argued that spatial uniformity can be improved by sonicating the same MB population with moderate acoustic pressures $(<200 \mathrm{kPa})$ and increasing their lifetime. Additionally, 5-cycle pulse sequences sustained cavitation for more than the longer 25-cycles sequences (figure 3). Thus shorter PLs offer the potential for greater lifetime of the flowing MBs, leading to a more uniform distribution of bioeffects along a vessel. This effect of short-PL sequences has been previously reported in vivo based on empirical evidence of BBB disruption (Choi et al 2011b, 2011a). In this paper we have confirmed that the reported improvement is directly correlated with the persisting MB-seeded acoustic cavitation activity (figures 3,6).

MB activity was shown to be maintained for approximately 40 pulses in moderate pressures for the PL of 25 cycles (figures 6 and 7), implying that the lifetime of MBs at a given pressure is directly correlated with the number of the experienced cycles. Within the stable cavitation regime, i.e. below 200kPa (figure 5), MBs are diminishing in size during each 
oscillation cycle due to rectified diffusion and dissolution of the encapsulated gas (Borden and Longo 2002), leading to a gradual decrease of echogenicity and cavitation activity (Radhakrishnan et al 2013). Thus, by defining the number of the emitted ultrasound cycles at a specific pressure, one can adjust the longevity of FUS-MBs interactions and control the spatiotemporal distribution of acoustic cavitation.

Our method for the distribution characterisation was implemented in vitro while assuming that the "idle" period between pulses would result in MB movement, though direct optical distance measurements were lacking. MB mobility was thus assessed by the calculated temporal constants $t_{50}$ and $t_{80}$. The highest $t_{80}$ values were found to be $\sim 30-35 \mathrm{~ms}$ (figure 3 ). Assuming that the MB velocity during the pulse-off time is approximately equal to the average fluid velocity of $10 \mathrm{~mm} / \mathrm{s}$, then a PRF of $1.25 \mathrm{kHz}$ (off-time of $0.75 \mathrm{~ms}$ ) and a PRP of $147 \mathrm{kPa}$, which has cavitation activity present for 40 pulses (figure 7), would result in individual MBs moving $\sim 0.3 \mathrm{~mm}$ along the lateral direction. The respective value for the continuous wave would be negligible due to primary radiation force effects (Dayton et al 2002). The pulse interval time scale ( 0.1-1ms for the various sequences) allowed inter-pulse MB movement of several microns $(1-10 \mu \mathrm{m})$ which is comparable with the MB size. It is possible that MBs could be "rolling" on the tubular or vascular wall, since these distances are on the same order as the $\mathrm{MB}$ radii. Increased $\mathrm{t}_{80}$ values are expected to lead to a more homogeneous cavitation distribution and bioeffect through the increased mobility of the cavitation nuclei. In our setting, the volume of the MB suspension within the focus is $0.35 \mathrm{~mm}^{3}$. For the clinical dose $\left(2 \cdot 10^{6} \mathrm{MBs} / \mathrm{ml}\right)$ and assuming uniform MB distribution across the $5 \mathrm{~mm}$ lateral FWHM, we would have $700 \mathrm{MBs}$ over $5 \mathrm{~mm}$ or $1 \mathrm{MB}$ every $7 \mu \mathrm{m}$. If each MB moved more than $7 \mu \mathrm{m}$ (which is the case with most of our pulse sequences), the spatial distribution of acoustic cavitation activity would be uniform throughout the focal volume. For the in vivo applications, the concept of "mobile" bubbles demonstrated here could change the cavitation-induced inhomogeneous pattern of permeability increase (Nhan et al 2013, Stieger et al 2007) into a more uniform pattern, as shown by Choi et al. (2011b, 2011a).

All the aforementioned calculations are valid only if fast bubble destruction is avoided, i.e. for stable cavitation behaviour. It is argued here that stable cavitation is the dominant cavitation mode below $200 \mathrm{kPa}$ (figures 5,6,7). Pressures in the $100-200 \mathrm{kPa}$ window present equal or higher values of the output parameter (figure 4). Those pulse sequences are therefore 
equally or more efficient in terms of uniformity of the spatiotemporal acoustic cavitation distribution.

\subsection{Limitations of the study}

The findings of the present study were based on the data obtained using a single-element

PCD. This method is an efficient and inexpensive tool to evaluate cavitation activity, but is neither able to extract spatial information beyond the focal volume of interrogation nor distinguish cavitation activity of regions within the focal volume. The present results thus would be further validated by other techniques, such as passive acoustic mapping (PAM), to directly assess the spatial distribution of bubble activity (Gyöngy and Coussios 2010), and high-speed camera optical observations. Additionally, detectability limits of the PCD method are correlated with the electronic noise floor. Our temporal constants (figure 3) are thus prone to underestimation, since a signal falling below the system's noise floor could persist for a longer period but would not be detected. Another limitation of PCD is that when spectral analysis is required, short PLs present an increased mixture of frequency content and therefore harmonics are inherently broadened. Consequently it was not possible to spectrally analyse the data for the 5-cycle pulse sequences as we did for the 25-cycles.

Furthermore, the bioeffect of such pulse shapes and sequences has to be estimated in future in vivo experiments. It is uncertain whether our pulse shapes and sequences will have an equally efficient in vivo bioeffect when compared with the existing ones. Our relatively simple physiologically relevant tube model provided insight about the effect of the various pulse sequences on the flowing bubbles cavitation dynamics. However, the real vasculature cannot be correctly approximated in vitro. A diverse range of parameters, such as vessel diameters and orientations, flow rates and acoustic pressure local inhomogeneity due to the interaction with the tissues are likely to affect the spatiotemporal distribution of cavitation dynamics. Vessel size for example is shown to affect the MB activity, especially in small-size vessels like the capillaries where the vessel wall directly influences the MB oscillation boundary conditions (Chen et al 2012, Caskey et al 2007). Additionally, water is not the ideal tissue-mimicking material since its acoustic properties significantly differ from the respective values of the tissues. Future studies are planned to be carried out in agar phantom models, in an effort to increase physiological relevance and adequately mimic in vivo conditions. Another source of bias is related with the MB concentration in each of the experiments. Whereas every effort was made to avoid concentration variations, it is likely that there were 
temporal fluctuation in the concentration of the sonicated MBs. Such fluctuation may be responsible for the unexpected results in the total emitted energy (figure 2) or the temporal constants of each sequence (figure 3). Finally, in vivo blood flow rates vary considerably, whereas in our proof-of-principle study only one set flow rate was used. To reinforce our findings regarding MB mobility, the effect of various flow rates will be investigated in future experiments using PAM. Nevertheless, our simple in vitro model can prove useful in creating safe and efficient ultrasound pulse sequences for use in every kind of therapeutic application.

\subsection{Clinical relevance}

Low power and short-PL sequences can be used for a wide range of clinical applications such as capillary opening techniques for targeted drug delivery, sonothrombolysis, and sonoporation. Short PLs with low amplitude offer the advantage of limited interference, reduced standing-wave formation and negligible thermal effects. Particularly for FUS-based therapies in the brain, the formation of standing waves within the skull is expected to be avoided when employing short PL therapeutic pulses. Furthermore, the focal volume can be more accurately defined since skull-specific aberrations and sidelobe magnitude could be easily ignored for short-PL sonication (Jones et al 2013, O'Reilly et al 2014). We have shown here that cavitation output for short 5-cycle pulse shapes is similar to longer 25-cycles shapes (figure 4). It is therefore expected that a shorter pulse is likely to have a similar bioeffect in the BBB disruption, as shown before (Choi et al 2011b, 2011a). Furthermore, it has been shown in vivo that single-cycle sonication at repetition periods similar to the present work can produce BBB disruption and successful drug delivery into the brain (O'Reilly et al 2011). Additionally, the spatial distribution of the drug concentration was found to be more uniform within the focal volume for sonication with short-PL and high PRF (Choi et al 2011b). The aforementioned in vivo studies reported the macroscopic bioeffects produced by short-PL sonication and in this work we provided a microscopic explanation based on MB mobility during the pulse off-time.

Sonothrombolysis is also likely to be favoured by the time-resolved cavitation activity of the flowing MBs. With known size and location of a thrombus in a vessel, our pulse sequences could be used to uniformly treat the entire pathology. The time constants found here (figure 3) could be employed to calculate the proper PL and inter-pulse interval that would allow MBs to travel and acoustically treat the whole length of the thrombus before reaching their fragmentation point. Furthermore, the proper selection of the acoustic pressure and the 
relative cavitation output (figures 2 and 4) could provide efficient and safe therapy, minimising damage-related high-amplitude inertial cavitation. Similar ideas and methodologies can be applied in other MB-mediated therapeutic applications.

\section{Conclusion}

5 In conclusion, we were able to incorporate the presence of flow in the control of the spatiotemporal distribution of acoustic cavitation activity by creating and testing new therapeutic pulse sequences, comprising of pulse trains with $\mu$ s pulse intervals. Stable cavitation with high acoustic cavitation output and increased persistence was noticed at pressures below $200 \mathrm{kPa}$ and for longer time intervals between the pulses. Shorter PLs allowed longer acoustic cavitation activity and MB mobility, favoured improved temporal distribution and presented increased acoustic output values. Low-power short-pulse sequences can thus uniformly distribute the MB-seeded acoustic cavitation activity and provide safe, efficient and homogenous treatment in a wide range of pathologies. Future work involves the investigation of the effect of flow rates and vessel diameters on the present pulse sequences efficacy. Finally, PAM will be used to determine the spatial distribution of acoustic cavitation activity.

\section{Acknowledgements}

The authors wish to acknowledge Dr. T. Matsunaga for the guidelines and discussions in the MB manufacturing process. Additionally, the authors wish to thank Dr. Mengxing Tang and Dr. Robert Eckersley for fruitful discussions and Mr. Gary Jones for building the experimental apparatuses. This work was funded by the Royal Society Research Grant 2014 (RG130795). A.N.P. wishes to thank the Bodossakis foundation for financial support during this study.

The authors declare no conflict of interest regarding the aims or results of the present paper. 


\section{References}

Apfel R 1997 Sonic effervescence: A tutorial on acoustic cavitation J. Acoust. Soc. Am. 101 1227-37

Apfel R E and Holland C K 1991 Gauging the likelihood of cavitation from short-pulse, low-duty cycle diagnostic ultrasound. Ultrasound Med. Biol. 17 179-85

5 Baseri B, Choi J J, Tung Y-S and Konofagou E E 2010 Multi-modality safety assessment of blood-brain barrier opening using focused ultrasound and definity microbubbles: a short-term study. Ultrasound Med. Biol. 36 1445-59

Borden M A and Longo M L 2002 Dissolution Behavior of Lipid Monolayer-Coated, Air-Filled Microbubbles: Effect of Lipid Hydrophobic Chain Length Langmuir 18 9225-33

10 Caskey C F, Stieger S M, Qin S, Dayton P a and Ferrara K W 2007 Direct observations of ultrasound microbubble contrast agent interaction with the microvessel wall. J. Acoust. Soc. Am. 122 1191-200

Chen H, Brayman A a and Matula T J 2012 Characteristic microvessel relaxation timescales associated with ultrasound-activated microbubbles. Appl. Phys. Lett. 101163704

Chen W-S, Brayman A A, Matula T J and Crum L A 2003 Inertial cavitation dose and hemolysis produced in vitro with or without Optison. Ultrasound Med. Biol. 29 725-37

Choi J and Coussios C 2012 Spatiotemporal evolution of cavitation dynamics exhibited by flowing microbubbles during ultrasound exposure J. Acoust. Soc. Am. 132

Choi J J, Carlisle R C, Coviello C, Seymour L and Coussios C-C 2014 Non-invasive and real-time passive acoustic mapping of ultrasound-mediated drug delivery. Phys. Med. Biol. 59 4861-77

Choi J J, Pernot M, Small S a and Konofagou E E 2007a Noninvasive, transcranial and localized opening of the blood-brain barrier using focused ultrasound in mice. Ultrasound Med. Biol. 33 95-104

Choi J J, Selert K, Gao Z, Samiotaki G, Baseri B and Konofagou E E 201 1a Noninvasive and localized bloodbrain barrier disruption using focused ultrasound can be achieved at short pulse lengths and low pulse repetition frequencies. J. Cereb. Blood Flow Metab. 31 725-37

Choi J J, Selert K, Vlachos F, Wong A and Konofagou E E 2011b Noninvasive and localized neuronal delivery using short ultrasonic pulses and microbubbles. Proc. Natl. Acad. Sci. U. S. A. 108 16539-44

Choi J J, Wang S, Tung Y-S, Morrison B and Konofagou E E 2010 Molecules of various pharmacologicallyrelevant sizes can cross the ultrasound-induced blood-brain barrier opening in vivo. Ultrasound Med. Biol. 36 58-67

30 Choi J, Pernot M, Brown T R, Small S a and Konofagou E E 2007b Spatio-temporal analysis of molecular delivery through the blood-brain barrier using focused ultrasound. Phys. Med. Biol. 52 5509-30

Chomas J E, Dayton P, May D and Ferrara K 2001 Threshold of fragmentation for ultrasonic contrast agents. $J$. Biomed. Opt. 6 141-50

Church C C 2005 Frequency, pulse length, and the mechanical index Acoust. Res. Lett. Online 6162

Church C and Carstensen E 2001 "Stable" inertial cavitation Ultrasound Med. Biol. 27 1435-7

Ciaravino V, Flynn H G and Miller M W 1981 Pulsed enhancement of acoustic cavitation: A postulated model Ultrasound Med. Biol. 7 159-66 
Daffertshofer M, Gass A, Ringleb P, Sitzer M, Sliwka U, Els T, Sedlaczek O, Koroshetz W J and Hennerici M G 2005 Transcranial low-frequency ultrasound-mediated thrombolysis in brain ischemia: increased risk of hemorrhage with combined ultrasound and tissue plasminogen activator: results of a phase II clinical trial. Stroke. 36 1441-6

5 Dayton P a., Allen J S and Ferrara K W 2002 The magnitude of radiation force on ultrasound contrast agents $J$. Acoust. Soc. Am. 1122183

Dayton P, Morgan K, Klibanov A, Brandenburger G, Nightingale K and Ferrara K 1997 A preliminary evaluation of the effects of primary and secondary radiation forces on acoustic contrast agents IEEE Trans. Ultrason. Ferroelectr. Freq. Control 44 1264-77

10 Deelman L E, Declèves A-E, Rychak J J and Sharma K 2010 Targeted renal therapies through microbubbles and ultrasound. Adv. Drug Deliv. Rev. 62 1369-77

Ferrara K, Pollard R and Borden M 2007 Ultrasound microbubble contrast agents: fundamentals and application to gene and drug delivery. Annu. Rev. Biomed. Eng. 9 415-47

Fischer K, McDannold N and Zhang Y 2009 Renal ultrafiltration changes induced by focused US Radiology 253 697-705

Flynn H G and Church C C 1984 A mechanism for the generation of cavitation maxima by pulsed ultrasound. $J$. Acoust. Soc. Am. 76 505-12

Graham S M, Carlisle R, Choi J J, Stevenson M, Shah A R, Myers R S, Fisher K, Peregrino M, Seymour L and Coussios C C 2014 Inertial cavitation to non-invasively trigger and monitor intratumoral release of drug from intravenously delivered liposomes J. Control. Release 178 101-7

Gruber M J, Bader K B and Holland C K 2014 Cavitation thresholds of contrast agents in an in vitro human clot model exposed to $120-\mathrm{kHz}$ ultrasound J. Acoust. Soc. Am. 135 646-53

Gyöngy M and Coussios C-C 2010 Passive cavitation mapping for localization and tracking of bubble dynamics. J. Acoust. Soc. Am. 128 EL175-80

Hosseinkhah N, Chen H, Matula T J, Burns P N and Hynynen K 2013 Mechanisms of microbubble-vessel interactions and induced stresses: a numerical study. J. Acoust. Soc. Am. 134 1875-85

Hosseinkhah N and Hynynen K 2012 A three-dimensional model of an ultrasound contrast agent gas bubble and its mechanical effects on microvessels. Phys. Med. Biol. 57 785-808

Hynynen K, McDannold N, Vykhodtseva N and Jolesz F 2001 Noninvasive MR Imaging-guided Focal Opening of the Blood-Brain Barrier in Rabbits 1 Radiology 220

Jensen C R, Ritchie R W, Gyöngy M, Collin J R T, Leslie T and Coussios C-C 2012 Spatiotemporal monitoring of high-intensity focused ultrasound therapy with passive acoustic mapping. Radiology 262 252-61

Jones R M, O’Reilly M a and Hynynen K 2013 Transcranial passive acoustic mapping with hemispherical sparse arrays using CT-based skull-specific aberration corrections: a simulation study. Phys. Med. Biol. 58 4981-5005

Konofagou E E 2012 Optimization of the ultrasound-induced blood-brain barrier opening. Theranostics 2 122337

Lauterborn W 1976 Numerical investigation of nonlinear oscillations of gas bubbles in liquids J. Acoust. Soc. Am. 59283 
Li P, Gao Y, Zhang J, Liu Z, Tan K, Hua X and Gong J 2013 Renal interstitial permeability changes induced by microbubble-enhanced diagnostic ultrasound. J. Drug Target. 21 507-14

Liu Y, Yi S, Zhang J, Fang Z, Zhou F, Jia W, Liu Z and Ye G 2013 Effect of microbubble-enhanced ultrasound on prostate permeability: a potential therapeutic method for prostate disease. Urology 81 921.e1-7

5 Marmottant P, van der Meer S, Emmer M, Versluis M, de Jong N, Hilgenfeldt S and Lohse D 2005 A model for large amplitude oscillations of coated bubbles accounting for buckling and rupture J. Acoust. Soc. Am. 118 3499

Nhan T, Burgess A, Cho E E, Stefanovic B, Lilge L and Hynynen K 2013 Drug delivery to the brain by focused ultrasound induced blood-brain barrier disruption: Quantitative evaluation of enhanced permeability of cerebral vasculature using two-photon microscopy. J. Control. Release 172 274-80

O'Reilly M A, Jones R and Hynynen K 2014 Three-Dimensional Transcranial Ultrasound Imaging of Microbubble Clouds Using a Sparse Hemispherical Array IEEE Trans. Biomed. Eng. 61 1285-94

O’Reilly M a., Waspe A C, Ganguly M and Hynynen K 2011 Focused-Ultrasound Disruption of the BloodBrain Barrier Using Closely-Timed Short Pulses: Influence of Sonication Parameters and Injection Rate Ultrasound Med. Biol. 37 587-94

O’Reilly M and Hynynen K 2012 Blood-Brain Barrier: Real-time Feedback-controlled Focused Ultrasound Disruption by Using an Acoustic Emissions-based Controller Radiology 263

Palanchon P, Tortoli P, Versluis M and de Jong N 2005 Optical observations of acoustical radiation force effects on individual air bubbles IEEE Trans. Ultrason. Ferroelectr. Freq. Control 52 104-10

Qin S and Ferrara K W 2006 Acoustic response of compliable microvessels containing ultrasound contrast agents. Phys. Med. Biol. 51 5065-88

Radhakrishnan K, Bader K B, Haworth K J, Kopechek J a, Raymond J L, Huang S-L, McPherson D D and Holland C K 2013 Relationship between cavitation and loss of echogenicity from ultrasound contrast agents. Phys. Med. Biol. 58 6541-63

De Saint Victor M, Crake C, Coussios C-C and Stride E 2014 Properties, characteristics and applications of microbubbles for sonothrombolysis. Expert Opin. Drug Deliv. 11 187-209

Sennoga C a, Mahue V, Loughran J, Casey J, Seddon J M, Tang M and Eckersley R J 2010 On sizing and counting of microbubbles using optical microscopy. Ultrasound Med. Biol. 36 2093-6

Stieger S, Caskey C and Adamson R 2007 Enhancement of Vascular Permeability with Low-Frequency Contrast-enhanced Ultrasound in the Chorioallantoic Membrane Model Radiology 243

Stride E P and Coussios C C 2010 Cavitation and contrast: the use of bubbles in ultrasound imaging and therapy Proc. Inst. Mech. Eng. Part H J. Eng. Med. 224 171-91

Tangelder G J, Slaaf D W, Muijtjens a. M, Arts T, oude Egbrink M G and Reneman R S 1986 Velocity profiles of blood platelets and red blood cells flowing in arterioles of the rabbit mesentery Circ. Res. 59 505-14

Tung Y-S, Vlachos F, Choi J J, Deffieux T, Selert K and Konofagou E E 2010 In vivo transcranial cavitation threshold detection during ultrasound-induced blood-brain barrier opening in mice. Phys. Med. Biol. 55 6141-55

Unger E C, Porter T, Culp W, Labell R, Matsunaga T and Zutshi R 2004 Therapeutic applications of lipidcoated microbubbles. Adv. Drug Deliv. Rev. 56 1291-314 
Vlachos F, Tung Y-S and Konofagou E 2011 Permeability dependence study of the focused ultrasound-induced blood-brain barrier opening at distinct pressures and microbubble diameters using DCE-MRI. Magn. Reson. Med. 66 821-30

Wang G, Zhuo Z, Xia H, Zhang Y, He Y, Tan W and Gao Y 2013 Investigation into the impact of diagnostic ultrasound with microbubbles on the capillary permeability of rat hepatomas. Ultrasound Med. Biol. 39 $628-37$

Wang S, Samiotaki G, Olumolade O, Feshitan J a and Konofagou E E 2014 Microbubble type and distribution dependence of focused ultrasound-induced blood-brain barrier opening. Ultrasound Med. Biol. 40 130-7

Yu H and Xu L 2014 Cell experimental studies on sonoporation: State of the art and remaining problems $J$. Control. Release 174 151-60

Zlokovic B V 2008 The blood-brain barrier in health and chronic neurodegenerative disorders. Neuron 57178 201 